\title{
Consideraciones sobre la categoría epistemológica de modelo
}

\author{
Adriana Patricia Gallego Torres*
}

Artículo recibido: 26-10-2005 y aprobado: 26-04-2006

\section{General considerations about model's epistemological category}

Resumen: En este artículo la autora presenta las diversas concepciones acerca de la categoría epistemológica de modelo científico. Intenta demostrar que esta categoría sigue estando afincada en el estatuto científico de la física, por lo que emplear la de teoría científica o la de modelo sin unas especificaciones desde aproximaciones históricas no aporta un cambio significativo en la mirada habitual sobre el estatuto científico de las diferentes ciencias de la naturaleza, especialmente si las conceptualizaciones al respecto siguen teniendo como matriz la física.Tampoco contribuye a una didáctica de la modelización o modelación, esto es, la de la construcción de modelos científicos en el aula, como se demuestra en los párrafos de este artículo.

Abstract: This article presents some conceptions regarding the epistemological category of the scientific model. It tries to demonstrate that this category is immersed in the scientific statute of Physics, due to it is not significant to use the category of scientific theory or the category of model without taking into considerations the historical foundations. This use does not provide a meaningful change in the overview for the scientific statute of the different sciences, and does not contribute to the Didactics of modelization.

Key words: Theory, scientific model, history, epistemology, didactics of modelization.

Palabras clave: Teoría, modelo científico, historia y epistemología, didáctica de la modelación.

* Doctora en Ciencias Físicas -Didáctica de las Ciencias- de la Universidad de Valencia, España. Profesora de la Pontificia Universidad Javeriana y de la Universidad Distrital Francisco José de Caldas de Bogotá, D.C., Colombia. Adriana.P.Gallego@uv.es

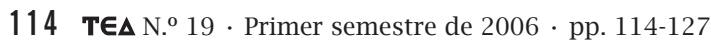




\section{Introducción}

Como es conocido por los especialistas, actualmente las investigaciones en didáctica de las ciencias de la naturaleza están ocupándose del problema de la modelación (modelización) en el aula, tanto con estudiantes de secundaria, como con profesores en formación inicial (Grosslight, Unger, Jay y Smith, 1991; Van Driel y Verloop, 1999; Justi, 2002; Crawford y Cullin, 2004). La mayoría de ellas se basan en la formulación que, al respecto de la categoría de modelo científico ha propuesto G. N. Giere (1990), sobre todo por sus tratamientos cognoscitivos. No obstante, se han elaborado ya críticas que, desde el recurso a una reconstrucción histórica de cada una de las ciencias de la naturaleza, hablan a favor de que la conceptualización de Giere no puede ser generalizada (Greca y Dos Santos, 2005); en otras palabras, y para emplear la categoría de T. S. Kuhn (1972), la constitución histórico-epistemológica de la física no puede seguir siendo el paradigma para examinar el estatuto científico de las otras ciencias de la naturaleza.

L. Gunter (1980) sostuvo que la categoría de modelo es frecuentemente empleada en las ciencias de la naturaleza y en las ciencias sociales, desde puntos de vista diferentes y a veces contradictorios, por lo que, de haber alguna semejanza entre las diferentes conceptualizaciones, es únicamente en lo general. Para este autor, superficialmente un modelo es toda clase de relaciones que se establecen entre un objeto o eventos originales y su correspondiente representación, la que puede ser entendida como un mapa en el sentido matemático de la expresión.
Esto ha dado origen a distinciones y clasificaciones de distintos tipos y usos de dicha categoría, que han ocupado mucho espacio en las revistas especializadas. La confusión creada es significativa, en virtud de que los diferentes autores han establecido distintas terminologías para aludir supuestamente a un mismo referente. No se requiere acudir a las ciencias sociales para demostrar que tal disparidad se sucede en las ciencias de la naturaleza, como se demostrará en las referencias bibliográficas que sustentan el presente artículo.

Ante el reto de formular y desarrollar una didáctica de la modelación o de la actividad de construir modelos científicos en el aula, se hace indispensable no sólo esclarecer en cada proyecto de investigación lo que en él se ha de entender por modelo científico, sino también precisar histórica y epistemológicamente la clase de modelo que ha dominado y ha sido aquel alrededor del cual cada comunidad ha desplegado sus mayores y mejores éxitos creativos y científicos. Es éste el objetivo que anima la discusión que aquí se concita.

Es indiscutible que hay razones para esta dominancia. La física, como la primera ciencia de la modernidad constituida como tal, se convirtió en ese paradigma a partir de los trabajos de Galileo y de su brillante culminación con la dinámica de Newton. La imagen mecanicista del mundo que instauró y el éxito predictivo logrado con la matematización e instrumentación de las relaciones entre causa y efecto hicieron que la ciencia de Newton adquiriera este estatuto. Cualquier otro campo de saber y de investigación que aspirara a ser considerado como científico tenía 
necesariamente que retomar los presupuestos de esta ciencia exitosa en sus predicciones. Ocurrió en la biología, en la que el corazón fue asimilado a una bomba neumática y las redes de circulación de la sangre a ductos, tanto como -cuando lo más complejo que se tenía era la central telefónica- el cerebro se asimiló a dicha central. En la sociología inicial ocurrió algo análogo, puesto que la sociedad se concibió como una maquinaria, de la que cada uno de los seres humanos se constituyó en una pieza de la misma (Buckley, 1970). No es extraño que estas metáforas continúen en la actualidad.

Para las correspondientes comparaciones histórico-epistemológicas entre la construcción de la física y las otras ciencias de la naturaleza, se pueden tomar como ejemplo la Principia y la Óptica de Newton, elaboradas desde un punto de vista euclidiano y siguiendo la estructura de los Elementos, por cuanto dicha estructuración inicial, para resumir, sigue la metodología de las definiciones, postulados, demostraciones geométricas y corolarios (Torres Assis, 1998). Destáquese, para reiterarlo, que detrás de la propuesta newtoniana, su sistema mecánico es análogo a un reloj, analogía a partir de la cual se asumió que dicho sistema seguía esquemas repetitivos y pautas discernibles, fundamento de su carácter predictivo.

Para una comprensión de la ciencia de Newton que hoy se hace objeto de trabajo didáctico en las aulas, es menester señalar que la Principia fue reescrita por J. L. Lagrange, en 1778 y P. S. Laplace, en 1796, de tal manera que los principios mecanicistas de Newton cambiaron radicalmente (Schneer, 1975). Podría sostenerse que la "mecánica racional", en esa reformulación matemática, dejó de lado la analogía del reloj, convirtiéndose en una estructura conceptual y metodológica de carácter simbólico.

Una comparación del libro de A. L. Lavoisier, Tratado elemental de química, de 1789, con la Principia y la Óptica de Newton, demuestra que la química, por ejemplo, se empezó a escribir con un lenguaje y una estructura metodológica diferentes. Este análisis repara también en el hecho de que la comunidad de los físicos de la época rechazaron y se burlaron de la propuesta icónica de modelo para el átomo (Schneer, 1975), presentada por J. Dalton, en su New System of Chemical Pilosophy, de 1827. Un examen de las mismas características al Origen de las especies, de C. Darwin, la conocida como "teoría de la evolución", tampoco obedece a los esquemas de los físicos.

A finales del siglo XIX y comienzos del siglo XX, se genera en la física una revolución científica que da origen a una revolución epistemológica, por cuanto se pone en tela de juicio la imagen de ciencia y de la actividad científica que venía imperando. En esta revolución epistemológica, desde la necesidad de las consecuencias que exigía, personajes de ciencias con inquietudes filosóficas elaboraron las propuestas que, de una manera u otra, han canalizado dichas perspectivas de análisis. En este contexto, se revisan las propuestas de K. Popper (1962), T. S. Kuhn (1972) e I. Lakatos (1983), para sólo mencionar los más referenciados. Se encuentra de nuevo que los tres autores se basan en los cambios a los que se ha aludido, a pesar de que Kuhn se ocupa de la quími- 
ca para ilustrar su concepción de cambio paradigmático.

La categoría epistemológica de la cual dan cuenta y constituye el fundamento de sus aproximaciones, es la de teoría, como un conjunto de proposiciones ligadas de manera deductiva. En Popper es evidente; Kuhn sostiene que es una teoría sustituta la que alcanza el estatuto de paradigma, después de que ha sido admitida por una comunidad científica dada; Lakatos propone como ejemplo de su núcleo firme las tres leyes de Newton y la de la gravitación universal. Se reafirma, entonces, que las aproximaciones epistemológicas para el establecimiento del estatuto científico de ciencias diferentes de la física quedaría suspendido, salvo que se admitieran otras definiciones acerca de teoría, incluso la del saber común y cotidiano, algo que no es rigurosamente admisible.

\section{La categoría de modelo científico}

Teniendo como punto temporal el siglo $\mathrm{XX}$, para la revisión de aquello que los distintos tratadistas han elaborado en torno a esta categoría epistemológica, hay que decir que M. Scheler (1936), desde una perspectiva kantiana, en su análisis de los fundamentos de la física emplea la de modelo lógico-mecánico, que se propone para dar cuenta de los fenómenos de la naturaleza, dada la necesidad de percibir y pensar estos fenómenos en términos de explicaciones mecánico- formales. Para este filósofo, dicho modelo parte de un pensamiento relacionante previamente explicitado, que hace posible la ordenación de estos fenómenos en un modelo. Tal procedimiento obedece a un orden legal que instaura el proceso de conocimiento y la experiencia arrojada por éste y no por un orden desconocido e íntimo de la naturaleza, producto de la simple y común observación. Los fenómenos objeto de modelación existen, para el investigador, precisamente porque ha propuesto para ellos un modelo lógico- mecánico.

Un análisis en profundidad de la concepción de M. Scheler conduce a ubicar su propuesta en el contexto de la física de su época, la que relacionó las ecuaciones de la mecánica con la de los fenómenos eléctricos, de tal manera que, teniendo en cuenta la admisible unidad de los objetos de saber y de investigación de los físicos, ellos han intentado siempre lograr una unificación, es decir, construir una teoría en la que todas las incógnitas del universo se resolverían.

T. S. Kuhn (1972) se refiere a la categoría de modelo en términos del paradigma dominante dentro de un colectivo de especialistas, que establece la concepción de ciencia y de práctica científica, en la que son formadas las nuevas generaciones de practicantes profesionales, productores de saber; es decir, habría una cierta connotación didáctica y pedagógica. La referencia a modelo en Kuhn, salvo mejores análisis, carece de un estatuto epistemológico. Para él, cada modelo recoge toda una tradición investigativa. I. Lakatos (1983) sostiene que la construcción de un modelo se deriva de la heurística positiva de cada programa de investigación. El modelo especifica el conjunto de condiciones iniciales que los enunciados legales del programa exigen y que se relacionan lógicamente en términos de proporcionalidad, con el fin de formular las predicciones empíricas, que, de cumplirse, apoyarán positivamente al 
correspondiente programa. De acuerdo con esta última especificación, un modelo científico es un intermediario entre la teoría que sustenta el programa y la praxis experimental.

N. R. Hanson (1958) es del parecer que un modelo es una estructura conceptual que sugiere un marco de ideas para un conjunto de descripciones, que se unen inferencialmente, y que de otra manera no serían sistematizables. Agrega que el modelo así concebido impulsa la inteligibilidad, ayuda a la comprensión de los fenómenos a los que hacen referencia las descripciones, proporcionando espacios de explicaciones posibles y direcciones del intelecto hacia lo no sorprendente. Una interpretación de la propuesta de este autor es aquella que afirma que todo modelo así elaborado difiere significativamente del referente de realidad o de naturaleza, para el cual se propone, ya que de no ser así, la estructura observacional de partida sería obvia para todo aquel que se enfrentase con las descripciones o, al menos, tan obvia como el modelo. Puntualiza que cada modelo es una manera de presentar las estructuras que posiblemente tengan las descripciones de la porción de realidad o de la naturaleza que se hacen objeto de estudio; en otras palabras, cualquier supuesto de correspondencia uno-a-uno entre modelo y descripciones concebidas como reales es una visión ingenua de la actividad científica.

Algunas elaboraciones recientes proponen que un modelo científico es una construcción supuesta y, por tanto, arbitraria, de un conjunto de objetos o procesos que se delimitan con miras a un estudio sistemático del comportamiento, provocado o no, de las interacciones de ese conjunto de objetos o procesos. En este orden de ideas, se establece que si el referente de realidad o de naturaleza es asumido desde los presupuestos de la dinámica de los sistemas no lineales, cualquier modelo es, en sí, incompleto (Castro, 1992). Al respecto, se toma distancia de esta calificación, ya que parece suponer que la actividad científica tendría como finalidad última la elaboración definitiva de modelos completos o, de otra manera, terminales para cada porción de realidad o de naturaleza. Como lo sostienen otros autores, para cada porción o sector son formulables distintos modelos, dependiendo esto de los esquemas de partida y de las intencionalidades de cada uno de los colectivos de especialistas.

Puesto que se ha hecho alusión al caso de la química, se destaca aquí los pocos análisis existentes en la literatura histórico-epistemológica, dedicados a reflexiones fundamentadas en torno al estatuto científico de esa ciencia, cuyos orígenes conceptuales y metodológicos pueden plantear y demostrar, mediante el recurso a la historia, que sus construcciones, como se ha sugerido, difieren de las de la física y de la biología, por ejemplo. Sin embargo, recientemente se ha abierto un espacio de discusión especializado, que recurre a la categoría epistemológica de modelo, para dar cuenta de un estatuto científico propio de la química, distinto del de la física. No obstante, las síntesis que se elaboran de algunos autores seleccionados sugieren que no avanzan en esta diferenciación necesaria.

J. Tomasi (1999) estipula que los modelos pueden ser clasificados como 
matemáticos y físicos, sin aportar nuevas consideraciones. Anota que todo modelo, de acuerdo con lo que ha significado en ciencias, es, por definición, incompleto. Los clasifica en modelos materiales y abstractos; estos últimos, afirma que se clasifican en icónicos, analógicos y simbólicos. Puntualiza que la praxis científica se basa en la formulación de modelos y de modelo de modelos. La pregunta que hay que hacerle a este tratadista, es la que indaga por: ¿Cuáles son los modelos propios de la química? ¿Qué distinciones habría que establecer entre éstos y los de las otras ciencias de la naturaleza?

G. Del Re (2000) establece que los modelos son simplificaciones o idealizaciones que, se supone existen en la naturaleza. Habla de modelos materiales y de modelos físicos, siendo estos últimos esenciales no sólo para describir sino, también, para conocer aquello que no es directamente accesible a los sentidos. Del Re cae igualmente en generalizaciones que son válidas para una introducción acerca de aquello que ha de entenderse por la categoría de modelo científico. No profundiza, por tanto, en interrogantes análogos a los formulados para J. Tomasi; es decir, parece que tampoco contribuye a una especificación del estatuto científico de la química, a partir de la categoría epistemológica de la cual trata.

E. F. Caldin (2002) puntualiza que los modelos son analogías de los sistemas reales y se reajustan de conformidad con las evidencias. Como se puede notarse queda sólo en el recurso a la figura de la analogía, que, como se sabe, es empleada en casi todos los campos explicativos, incluido, hasta cierto punto, el del saber común y cotidiano. ¿De qué manera los modelos científicos son analógicos o analogías de sistemas reales? ¿Qué es lo que caracteriza las analogías a las que acuden las comunidades de especialistas? ¿Desde qué razones históricas dar cuenta de tales analogías? ¿Cómo aparece la también categoría de sistema? ¿Desde dónde se supone que en la porción de realidad o de naturaleza que se hace objeto de estudio es un sistema?

H. L. Kretzenbacher (2003), desde la distinción que establece entre modelos científicos y metáforas, presenta en su disquisición, por un lado, que los modelos permiten enlazar teorías con observaciones empíricas y serían el recurso conceptual y metodológico que posibilita la aplicación de las teorías a clases especiales de objetos o de fenómenos. Por otro, que el hecho de que los modelos representen entidades físicas es lo que hace que a partir de ellos sea posible validar empíricamente las proposiciones. Este autor introduce la figura de la metáfora y establece acerca de ella que no toda metáfora es científicamente admisible. Es problemático, en razón de que la distinción entre metáforas y analogías no resulta conceptual y metodológicamente sostenible, sobre todo en el caso de las ciencias de la naturaleza; todo sin desconocer que el recurso a la figura de la metáfora es, relativamente, de vieja consideración (Howard, 1989).

Cabe resaltar que estos cuatro autores se ocupan de precisar la categoría de modelo científico desde la química. Sin embargo, la mayoría de ellos no estipulan cuál es el modelo que mejor responde a la construcción histórica de esta ciencia. Se quedan en las generalizaciones acerca de esta categoría, y pa- 
recen seguir en el paradigma de la física, para, como se ha establecido, afincar el estatuto de cientificidad. Kretzenbacher, por ejemplo, se ubica en la misma propuesta de Lakatos, esto es, cada modelo científico se elabora para contrastar las hipótesis derivadas de una teoría o, de otra manera y siguiendo a Popper, el modelo se deriva de las proposiciones observacionales de la teoría.

Algo semejante habría que sostener con respecto a O. Lombardi (1998), autora que, igualmente desde la física, entra a precisar aquello que se ha establecido en cuanto a la categoría de modelo en matemática. Puntualiza que los especialistas de esta disciplina se ocupan de los sistemas axiomáticos o conjunto de fórmulas relacionadas deductivamente mediante una lógica subyacente. El modelo matemático se crea cuando se procede a darle una interpretación al sistema, asignándole a cada término una referencia que pertenece a un dominio previamente establecido. Esta especialista aclara que toda teoría científica tiene como referente, no al sistema real o natural del cual pretende dar cuenta descriptiva y explicativamente, sino al modelo que se elabora para tal sistema. De la misma manera, afirma que una teoría en física puede ser despojada de su contenido semántico, para convertirla en un sistema axiomático.

Anótese que A. Badiou (1972), a partir de una posición marxista, estipula que un modelo en matemática es un haz de hipótesis al que se le supone relativamente completo y cuya coherencia y posterior desarrollo deductivo quedan garantizados por una codificación dentro del lenguaje de esta disciplina. Cita el teorema de Gödel-Henkin, en el que una teoría es coherente si y sólo si tiene un modelo. En este sentido, acude igualmente al teorema de Lowenheim-Henkin, que postula que una teoría formal que admite un modelo infinito, necesariamente admite un modelo numerable.

Es interesante recuperar la cita que Badiou presenta de G. Bachelard:

El modelo planetario de Bohr sólo hizo entrega de una imagen útil del átomo en la época en que la microfísica acompañaba la borradura de sus órbitas y, finalmente, la renuncia de esa imagen, en beneficio de un modelo estadístico. Quien no sabía renunciar al modelo, renunciaba al saber: toda detención en un modelo conforma un obstáculo epistemológico ¿Hasta qué punto el modelo permanece al margen de la producción de conocimiento? Con todo, en ese lugar no es recusable, no presenta siquiera un problema.

La anotación de Bachelard citada por Badiou, se inscribe en la convicción de que cualquier modelo que se elabora de una porción o aspecto de la realidad o de la naturaleza, que un colectivo haga objeto de saber, de investigación, de intervención, regulación y transformación, históricamente analizado es tan solo una de las diferentes maneras que los seres humanos optan por darle sentido a su vida. En el caso de las ciencias de la naturaleza, esta opción se traduce en un cambio, si cada proyecto educativo lo posibilita, en la concepción de mundo y del papel del ser humano en ese mundo.

A manera de síntesis es necesario sostener que no todas las ciencias de la naturaleza han seguido en su construcción histórica el proceso de geometrización y matematización de sus 
objetos de conocimiento, puesto que su complejidad no lo hace posible; es decir, no son reducibles a masas puntuales y, por tanto, a describir sus trayectorias en términos lineales, para las formulaciones deductivas en sistemas axiomáticos. Que los éxitos descriptivos y explicativos, tanto como los de sus intervenciones en la porción de realidad o de naturaleza de la cual dan cuenta, hablan a favor de la admisibilidad de sus discursos, hasta el punto de que todos los seres humanos, como es el caso de la medicina, se ponen en sus manos. Que todas las ciencias de la naturaleza retoman las construcciones conceptuales, metodológicas y tecnológicas de las otras, con miras a sus propósitos de saber y de investigación, sin que ello signifique una dependencia o sometimiento al estatuto científico de esas otras. Cada ciencia de la naturaleza está caracterizada por una historia propia, por un lenguaje específico, derivado de las intencionalidades de saber y de investigación de la comunidad de especialistas.
Como cada porción de realidad o de naturaleza es compleja, el ser humano y en particular las comunidades de especialistas que se dedican a elaborar sistemas y modelos han de partir del convencimiento de que esos sistemas y modelos tan sólo son aproximaciones, por lo que cualquiera aspiración a la verdad es una ilusión. Tanto K. Popper (1962) como T. S. Kuhn (1972) establecieron que la aspiración a la consecución de la verdad definitiva acerca de la constitución y el sentido del universo era una preocupación metafísica, tan válida como se quiera, y que la actividad científica vuelve al principio con cada cambio paradigmático.

\section{Una aportación}

Después de revisadas las diferentes conceptualizaciones en torno a la categoría de modelo científico, se presenta un esquema que es el punto de partida para someter a consideración de la comunidad de especialistas las aportaciones que en este artículo se asumen:

$$
\text { (Realidad / Naturaleza) } \leftrightarrow \text { Sistema Modelo } \leftrightarrow\left\{\begin{array}{l}
\text { Icónico (gráfico) } \\
\text { Analógico } \\
\text { Simbólico }
\end{array}\right.
$$

Se puede afirmar, entonces, que un modelo científico es una estructura conceptual y metodológica mediante la cual una comunidad de especialista da cuenta descriptiva y explicativamente de las características e interacciones que le ha atribuido a un sistema, sistema que ha construido como objeto de saber y de investigación. Ese sistema se supone que es de alguna manera equivalente ( $\equiv)$ o recoge en su conformación, como hi- pótesis contrastable, aquella parte de la realidad o de la naturaleza, de la cual se afirma que tal sistema es una representación admisible. Esto significa que de dicha porción es factible elaborar tantos sistemas cuantas sean las concepciones e intencionalidades de saber y de investigación que toman rigurosamente como punto de partida el respectivo grupo de especialistas. 
En consecuencia, es el sistema elaborado sobre el cual se dirigen todos los esfuerzos de conceptualización del colectivo, desde el convencimiento de que ese sistema no es la porción de realidad o de naturaleza sobre la cual enfoca sus intencionalidades de comprensión, ordenamiento, regulación e intervención. De ser así, los diseños experimentales para las consecuentes contrastaciones no se de dirigen a verificar si esa porción de realidad o de naturaleza se comporta como se concibe, por cuanto el interés focal se centra en el sistema, como un constructo a partir del cual aquello que se ha concebido acerca de dicha porción responde empíricamente a los supuestos de partida que se hallan en la base de la conversión de una parte de la realidad o de la naturaleza, en un sistema objeto de saber y de investigación.

Hay que recalcar que todo sistema es una especie de rejilla, en la que solo se incorporan aquellos elementos que el colectivo de investigadores considera relevantes para elaborar un cuadro suficientemente completo de la porción de realidad o de naturaleza a la que aspira dominar, regular y transformar mediante su conocimiento. Los puntos o nodos de la rejilla pueden ser abstracciones de objetos materiales o conceptos científicos cualitativos o métricos (Mosterín, 1978) y las relaciones entre esos nodos pensadas como interacciones mediante las cuales se intercambia energía o masa inercial; tales interacciones delimitadas en términos de hipótesis definirían propiedades físicas o químicas, a la vez que también establecerían cuál sería el comportamiento global del sistema.

Cada sistema es solidario dialéctica, conceptual y metodológicamente con un modelo. Entre cada sistema y el modelo correspondiente que se propone, existe una relación que es determinada por las concepciones iniciales desde las cuales cada grupo formula el sistema que se corresponde con las intencionalidades de saber y de investigación que se propone. En la medida en que ese grupo examina la adecuación y las concepciones de partida, éstas pueden entrar en un proceso de reelaboración paulatina, de tal manera que ese sistema posibilite la formulación de modelo que para, después de los respectivos análisis críticos, el colectivo tome la determinación de que ese sistema permite un modelo admisible que da cuenta descriptiva y explicativamente del sistema propuesto. El modelo en cuestión, al explicitar las interacciones y basarse en las hipótesis del comportamiento del sistema, será de carácter hipotético-deductivo. Los resultados específicos que se obtengan en los experimentos que se diseñen y realicen, lo contrastarán positiva o negativamente. En este sentido, y de conformidad con las contrastaciones positivas, el colectivo concluiría, tentativamente, que el modelo del y el sistema mismo, son admisibles.

Si se recuerda la relación entre las ciencias experimentales y los saberes tecnológicos, introducida por Galileo, además de resultados en términos de las interpretaciones de los valores arrojados por el tramado instrumental y por cada uno de los instrumentos del diseño experimental, es menester sostener que esa contrastación positiva hay que referirla a la también producción de nuevos tecnofactos, como a la de nuevos materiales. En este sentido, no sería la porción de realidad o de naturaleza el 
espacio de actuación, sino el sistema o rejilla que, por la delimitación conceptual y metodológica que se ha llevado a cabo, se aproxima a una representación tecnológica.

Hay que recordar aquí que los tratadistas, como lo demuestran las referencias bibliográficas en las que se sustenta el presente artículo, clasifican los modelos científicos en materiales y abstractos; para estos últimos establecen una taxonomía de icónicos (gráficos), analógicos y simbólicos. Se es del parecer de que, de acuerdo con esta taxonomía, ella en la especificidad de la formulación histórica de los modelos que caracterizan a cada una de las ciencias de la naturaleza inciden, primero, las concepciones de partidas, en el contexto de las formulaciones que -desde una perspectiva que considera podría ser fructífera- la comunidad correspondiente está examinando, en términos de lo no sorprendente, en principio.

Segundo, con la porción de realidad o de naturaleza que se convierte en sistema para ser objeto de estudio y de investigación. Será icónico o gráfico, si esa porción de realidad o de naturaleza no es directamente accesible a los sentidos, como condición empiropositivista discutible, o mediada por registros instrumentales, lo que significa una instrumentación de esa porción de realidad o de naturaleza objeto de saber y de investigación, que, en principio, no es la realidad ni la naturaleza. El modelo será icónico o gráfico, si esa porción no es directamente accesible a una observación primera, no mediada instrumentalmente; una no mediación en virtud de que el estado de la investigación tecnológica no ha diseñado y construido los instrumen- tos requeridos. Es el caso histórico, por ejemplo del modelo químico del átomo propuesto por J. Dalton, transformado posteriormente por la hipótesis de A. Avogadro y los trabajos de A. Laurent, C. Gerhardt, E. Frankland y A. Kekule, en el siglo XIX.

Afírmese que el modelo icónico que los químicos fueron construyendo paulatinamente para representar la estructura molecular de los materiales tuvo su contrastación positiva, primero con la síntesis de la urea y después con la de los azúcares existentes en la naturaleza, lo que les indicó que era admisible. Luego esa contrastación positiva se reforzó con la síntesis de la primera sustancia no existente en la naturaleza, la baquelita, a la que le siguió la de la sacarina, un material con un poder edulcorante 500 veces superior al del azúcar de caña, sin ser un azúcar. Le seguirá el primer medicamento: el salvarsán. Lo demás, para repetir la conocida frase, es historia.

Lo que demuestra el resumen que se condensa en el párrafo anterior, es lo ya sostenido en cuanto al poder de intervención sobre el sistema delimitado, que puede llevar consigo un modelo icónico, a la vez que, igualmente y desde sus especificidades, puede ser de carácter hipotético-deductivo, puesto que a partir de él es factible diseñar y fabricar, como es el caso anotado, materiales totalmente artificiales y, en consecuencia, construir espacios de producción en los que no se trata de la naturaleza, sino de una actividad con poder creativo.

La autora de este artículo reconoce su ignorancia en relación con la biología, a pesar de que es consciente de que el estatuto científico de esta ciencia no 
puede ser justificado por sus recursos actuales a la física y a la química, lo que podría ser catalogado, para este caso particular, como un discutible problema epistemológico. Se insiste en que el estatuto científico de esta ciencia es de otro tipo, aun cuando en este artículo se reconozca y no se ensaye un discurso al respecto.

El modelo será analógico cuando esas concepciones de partida tienen como fundamento un constructo técnico o tecnológico, mediante el cual se establece que las características e interacciones pueden ser representadas idealizando las que son propias de ese constructo. Podría señalarse al respecto la "mesa de billar" para el modelo cinético molecular de los gases, la máquina térmica de Carnot y su empleo de la cascada de agua para explicar los flujos de calor entre dos manantiales térmicos, y el oscilador armónico simple en el caso de la mecánica ondulatoria. Lo que de nuevo se recalca aquí es esa relación que desde Galileo se estableció entre las ciencias de la naturaleza y los saberes tecnológicos.

Anótese que en la delimitación del sistema o de la rejilla, cuando los puntos o nodos se plantean como conceptos métricos y las interacciones como intercambio de energía o de masa inercial, la construcción del modelo analógico está precedida de una geometrización de las interacciones referidas a aquello a lo que se hace referencia con cada concepto, como también a las interacciones entre los ámbitos de referencia de todos los conceptos implicados. Esa geometrización es la que posibilita que un modelo analógico pueda tener como base o ser expresado en un sistema axiológico.

El modelo es simbólico cuando se reformula el analógico. Acudiendo a la máquina ideal de Carnot y a los tratamientos posteriores de Clayperon, Kelvin y Clausius para el ejemplo de la construcción de la termodinámica clásica, esa máquina ideal inicial pierde cualquier referencia concreta, en virtud de que la estructura conceptual y metodológica se transforma para ser un conjunto de expresiones matemáticas en términos de ecuaciones diferenciales lineales, en las que se recogen las leyes que gobiernan las transformaciones energéticas, caloríficas, del trabajo termodinámico y de la entropía. Agréguense a lo anterior las funciones de trabajo de Gibas y de Helmholtz. En estas ecuaciones, y por tratamientos puramente matemáticos, se definen los conceptos de entalpía, de las capacidades caloríficas a presión y a volumen constantes, respectivamente.

Los modelos simbólicos serían caracterizables por su semejanza con los sistema axiomáticos de la matemática, en la medida en que integrarían sistema y modelo en una unidad mucho más estrecha de la que se recoge con la afirmación de unas relaciones dialécticas entre ambos. En este sentido, la formulación podría comenzar con una estipulación ontológica, vale decir: "Sea un sistema conformado por...", caso este en el que, si bien se acude a la clase de analogías aquí señaladas, el desarrollo deductivo de las proposiciones daría pie para hipótesis cuya contrastación quedaría en el dominio de los famosos "experimentos mentales" de Einstein. 


\section{Apuntes para una didáctica de la modelación}

Plantear la posibilidad de este campo de investigación en la nueva didáctica de las ciencias de la naturaleza exige de los didactas de las ciencias una ubicación conceptual y metodológica que la demuestre, con referencias bibliográficas tomadas de revistas especializadas en la nueva didáctica de las ciencias. Ello significa tomar distancia del también discurso de esa pedagogía, de generalidades, en el que cualquier opinión, sin importar sus factibles apoyos investigativos, es, de por sí válido; con el debido respeto, cualquier opinión, venga de donde venga, per se es válida. $\mathrm{Al}$ respecto, la autora de este artículo, cuyo resumen de tesis fue publicado en una revista especializada, se halla en condiciones, dados también los artículos que ha publicado en revistas especializadas.

Quiero puntualizar en torno al programa de doctorado que seguí y desde el cual me doctoré. Mi tesis doctoral Cum laude me habilita para sostener un discurso cuyo resumen está publicado en Enseñanza de las Ciencias.

1. Un discurso fundamentado en referencias bibliográficas, sobre la constitución, en general, de la ciencia que se quiere hacer objeto de trabajo en el aula y, particularmente, de cada uno de los modelos científicos que con propósitos didácticos integra y correlaciona con miras a que los estudiantes elaboren una imagen de ciencia, de científico y de la actividad profesional que caracteriza a estos especialistas.

2. Haber elaborado una explicación acerca de las razones por las cuales a lo largo de su versión histórica cada uno de los modelos que hace objeto de trabajo en el aula fue admitido, transformado y sustituido por otro. Desde esta perspectiva, identificar, caracterizar y categorizar los modelos que integra y correlaciona.

3. Puntualizar en que los estudiantes ingresan en el proceso con concepciones o ideas alternativas acerca de cada modelo, incluyendo, en principio, que nunca antes han sido sometidos a la experiencia didáctica de construir modelos científicos; todo porque provienen de una enseñanza de las ciencias de carácter tecnicista, esto es, reducida a definiciones, fórmulas matemáticas y aplicación de las mismas a los ejercicios de lápiz y papel.

4. Que es preciso que quien se comprometa con una didáctica de la modelación delimite claramente aquello que concibe como modelo científico, para desde allí establecer las diferencias necesarias entre las diversas ciencias de la naturaleza; diferencias que exigirían tratamientos didácticos distintos.

5. Una superación de la reducción tecnicista, para examinar en el aula cada modelo científico, a partir de las consecuencias que tuvo en las concepciones de mundo y las relaciones entre los seres humanos de cada época.

\section{A manera de conclusión}

Una vez realizada la revisión acerca de las distintas versiones existentes sobre la categoría de modelo científico, de la necesidad de que sea factible una didáctica de la modelación o de la modeliza- 
ción, la autora del presente artículo es del parecer de que:

- En primer lugar, hay tantas concepciones acerca de la categoría de modelo, cuantos especialistas se ocupan de ella.

- Que los trabajos de precisión conceptual y metodológica proceden de los físicos, basados en los éxitos de esta comunidad de especialistas.

- Que estas elaboraciones no pueden ser aplicadas a las otras ciencias de la naturaleza, como es el caso de la química, cuya construcción histórica no ha obedecido ni seguido los mismos parámetros conceptuales y metodológicos, dada la especificidad del objeto de saber y de investigación que se fijó la comunidad.

- Queda un interrogante en suspenso: ¿Cubren los modelos icónicos, analógicos y simbólicos el espectro suficiente para dar cuenta de la construcción histórica de la biología como una de las ciencias de la naturaleza? ¿Es preciso un taxón adicional?

\section{Bibliografía}

Badiou, A. (1972). El concepto de modelo. Bases para una epistemología materialista de las matemáticas. México: Siglo XXI.

Buckley, W. (1970). La sociología y la teoría moderna de los sistemas. Buenos Aires: Amorrortu.

Caldin, E. F. (2001). "The structure of chemistry in relation to the philosophy of science". International Journal for Philosophy of Chemistry, Vol. 8, $\mathrm{n}^{\circ} 2$, 103-121. En línea http://.hyle.org/journal/issues/8-2/caldin.html

Castro, E. A. (1992). "El empleo de los modelos en la enseñanza de la química”. Enseñanza de las Ciencias, 19(1), 73-79.

Crawford, B. A. y Cullin, M. J. (2004). "Supporting prospective teachers' conceptions of modeling in science". International Journal of Science Education, Vol. 26, nº. 11, 1.379-1.401.

Del Re, G. (2000). "Models and analogies in science". International Journal for Philosophy of Chemistry, Vol. 6, $\mathrm{n}^{\circ} 1$, 5-15.

En línea http://.hyle.org/journal/issues/6-1/delre.html

Giere, R. N. (1990). Explaining Science. Chicago: University of Chicago Press.

Grosslight, L., Unger, C., Jay, E. y Smith, C. (1991). "Understanding Models and their use in science conceptions of middle and high school teachers and experts". Journal of Research in Science Teaching, 28(9), 799-882.

Greca, I. M. y Dos Santos, F. M. T. (2005). Dificuldades da generalização das estratégias de modelação em ciências: O caso da física e da química. Investigações em Ensino de Ciências. En línea: http://if.ufrgs.br/public/ensino/vol10/n1/v10_n1_a2.htm

Gunter, L, (1980). Models in phisics: some pedagogical reflections based on the history of science. European Journal of Science Education, vol. 2, N. ${ }^{\circ} 1$, 15-23.

Hanson, N. R. (1958). Observation and explication: A guide to philosophy of science. Patterns of discovery. An in- 
query into the conceptual foundations of science. Cambridge: Cambridge University Press.

Howard, R. (1989). "Teaching sciences with metaphors”. SER, 70(252), 100103.

Justi, R. S. (2002). "Modeling Teachers' views on the Nature of modeling and implications for the Education of modelers". International Journal of Science Education, Vol. 24, $\mathrm{n}^{\circ} 4$, 369-387.

Kretzenbacher, H. L. (2003). "The aesthetics and heuristics of analogy. Model and metaphor in chemical communication". International Journal for Philosophy of Chemistry, Vol. 9, $\mathrm{n}^{\circ}$ 2, 191-218. En línea http://.hyle. org/journal/issues/9-2/kretzenbacher.html

Kuhn, T. S. (1972). La estructura de las revoluciones científicas. México: Fondo de Cultura Económica.

Lakatos, I. (1983). La metodología de los programas de investigación científica. Madrid: Alianza.

Lind, G. (1980). "Models in physics: Some pedagogical reflections base don the history of science". Europea Journal of Science Education, Vol. 2, $\mathrm{n}^{\circ} 1$, 15-23.
Lombardi, O. (1998). "La noción de modelo en ciencias”. Educación en Ciencias, Vol. II, n ${ }^{\circ} 4,5-13$.

Mosterín, J. (1978). "La estructura de los conceptos científicos”. Investigación y Ciencia, $\mathrm{n}^{\circ}$ 16, 82-93.

Popper, K. (1962). La lógica de la investigación científica. Madrid: Tecnos.

Scheler, M. (1936). Erkenntnis und Arbait. Leipzig: Der neue Geist.

Schneer, C. J. (1975). Mente y material. Barcelona: Bruguera.

Tomasi, J. (1999). “Towards chemical congruence of de model in theoretical chemistry". International Journal for Philosophy of Chemistry, Vol. 5, n ${ }^{\circ} 2$, 79-115. En línea http://.hyle.org/journal/issues/5-2/tomasi.html

Torres Assis, A. K. (1998). "Newton e suas grandes obras: o Principia e o Óptica”. En: De Almeida, M. J. P. M. e da Silva, H. C. (orgs.); Linguagens, leituras e ensino da ciência, Campinas: Unicamp, pp 37-52.

Van Driel, R. S. and Verloop, N. (1999). “Teachers' knowledge of models and modeling in science". International Journal of Science Education, 21(11), 1.141- 1.153 . 